
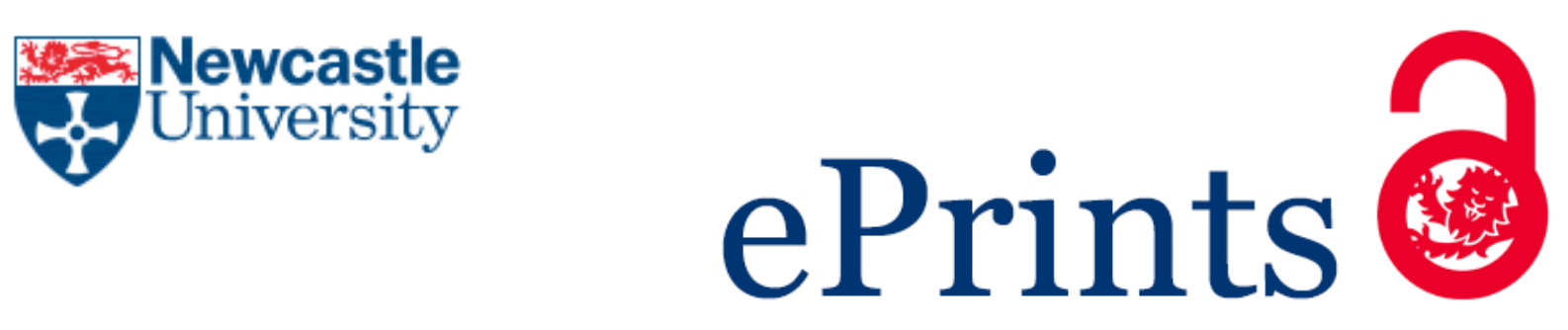

Bull SJ. Elastic properties of multilayer oxide coatings on float glass. Vacuum 2014, 114, 150-157.

\title{
Copyright:
}

Copyright @ 2015 Elsevier Ltd.

DOI link to article:

http://dx.doi.org/10.1016/j.vacuum.2014.12.012

Date deposited:

$15 / 12 / 2014$

Embargo release date:

19 December 2015

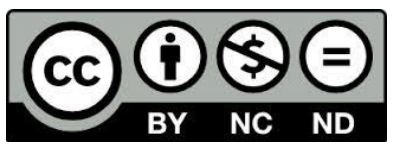

This work is licensed under a

Creative Commons Attribution-NonCommercial-NoDerivs 3.0 Unported License 


\section{Elastic properties of multilayer oxide coatings on float glass}

S J Bull

School of Chemical Engineering and Advanced Materials

Newcastle University

Newcastle upon Tyne

NE1 7RU, UK.

e-mail: steve.bull@ncl.ac.uk

\section{Abstract}

The damage resistance of multilayer oxide coatings on glass used for optical applications often depends on contact damage and the stress generation and relaxation mechanisms during coating deposition and subsequent application. Whereas it is relatively easy to design the optical properties of a multilayer coating to meet a particular specification it is more difficult to design its mechanical response. In part this is due to a lack of reliable mechanical property data for design, chief of which is the elastic modulus of each coating layer in the multilayer stack. Indentation tests can be used to determine the elastic and plastic properties of relatively thick $(>1 \mu \mathrm{m})$ coatings but, whereas it is possible to measure the plastic response of submicron coatings provided the indenter penetration is less than $10 \%$ of the coating thickness this is not the case for elastic properties where a much lower penetration is required. In this study the use of nanoindentation testing in conjunction with a simple modelling approach to determine suitable elastic properties of individual oxide layers in a multilayer coating stack is assessed. It is demonstrated that the ISO14577 extrapolation approach underestimates the contact moduli for $200 \mathrm{~nm}$ thick coatings but a multilayer model can be used to determine more realistic properties. Variations in elastic properties of the glass substrate in the near-surface region need to be taken into consideration to get good data from the model.

\section{Introduction}

Multilayer oxide coatings are used in a wide range of optical applications such as anti-reflection [1] and solar control coatings [2]. For instance in a typical solar control coating the active $10 \mathrm{~nm}$ thick silver layer is surrounded by antireflection coatings such as zinc oxide or tin oxide. These layers are also surrounded by barrier layers such as TiOxNy to prepare the glass substrate for the coatings and protect the coating surface from mechanical or chemical damage. All of these oxide coatings are around $10 \mathrm{~nm}$ thick and have been selected for their optical performance.

In service the main damage modes may depend on mechanical contact of the coating which can arise during handling of the coated glass, assembly into devices or in subsequent service [3]. Mechanical design is often secondary to optical design because it does not usually affect the primary function of the device. For good mechanical design the elastic properties of the coatings in the optical stack are often required but these are very difficult to measure in the thin coatings which are required for optical designs. In this study the possibility of using indentation measurements made on relatively thick coatings in conjunction with a simple modelling approach [4] to assess the validity of the properties for designs with thinner coatings is investigated. 
Nanoindentation has been used to measure the elastic and plastic properties of thin coatings $(\sim 1 \mu \mathrm{m})$ for some time now and methods to extract the properties of the coating from data for the coating/substrate system have been developed for single layer coatings [e.g. 5, 6]. This is most developed for plastic properties such as hardness where it is often assumed that if the indenter penetration is less than $10 \%$ of the coating thickness the hardness of the coating may be measured independent of the substrate. For elastic properties the required indenter penetration to measure the elastic response of the coating is much smaller, typically less than $1 \%$ of the coating thickness and this is almost impossible to achieve for most coatings. To deal with this issue the ISO14577 Part 4 standard [7] recommends extrapolating the variation of hardness or elastic modulus to zero depth to get a value for the properties of the coating. This method works reasonably well for coatings on a stiff substrate but gives considerable errors when the substrate is much more compliant than the coating [4].

For multilayer coatings measurements made on cross sections may be used to determine individual layer properties [8]. However, for anisotropic materials the properties measured from the cross section may be very different from those measured normal to the surface due to the effects of texture and grain size/shape [9]. Furthermore, as coatings become thinner the difficulty of confining stresses in an individual layer means that composite behaviour is often measured. Thus modelling the properties of the coating substrate system to extract the properties of an individual coating layer is the most useful approach.

Whereas there are a number of approaches that have been used to model the plastic properties of a multilayer coating during indentation [e.g. 4, 6] the modelling of the elastic response is less well developed [e.g. 10]. In this study a simple analytic model for the contact modulus of a coating/substrate system has been extended to multilayer coatings and the results used to determine reliable elasticity data for oxide coatings on glass that may be used in the design of solar control coatings.

\section{Experimental}

\subsection{Coatings investigated}

Oxide coatings which are the most significant layers in multilayer solar control coatings for architectural glass were deposited onto the air side of $4.2 \mathrm{~mm}$ thick float glass samples using typical commercial process parameters at the Pilkington Research Centre in Lathom, UK. To ensure that the properties of the coatings were typical each coating was deposited onto the same underlying structure made by depositing the lower layers in the multilayer with the same thickness and process parameters as in a commercial solar control coating design. The top layer was deposited to the same thickness as in the solar control coating but also to a thickness of $200 \mathrm{~nm}$ to enable indentation measurement. Layer materials and thicknesses are summarised in Table 1.

The coatings were produced by reactive sputtering from metallic targets using an oxygen backfill to generate stoichiometric coatings. Large glass sheets $(20 \mathrm{~cm}$ by $30 \mathrm{~cm})$ were coated in a linear vacuum chamber where they were moved past fixed magnetrons at a constant rate. Each coating was produced by a different magnetron cathode in the same coating chamber. Samples were then cut into $10 \mathrm{~cm}$ by $10 \mathrm{~cm}$ squares for indentation analysis. In these coatings a $10 \mathrm{~nm}$ silver film is the active solar control layer but this is protected from oxidation during deposition of subsequent oxide layers 
by a $5 \mathrm{~nm}$ zirconium overlayer; neither silver nor zirconium could be deposited to $200 \mathrm{~nm}$ thickness without significant oxidation on removal from the vacuum chamber. For this reason handbook values for elastic properties of these layers have been used in the modelling undertaken in this study [11].

The thickness of the individual layers was controlled by the magnetron power and translation speed and was confirmed by ellipsometry.

Table 1: Layer structure and thickness of the coatings deposited

\begin{tabular}{|l|l|l|l|l|l|l|}
\hline Sample & $\begin{array}{l}\text { TiOxNy } \\
\text { underlayer }\end{array}$ & ZnO & $\mathrm{Ag}$ & $\mathrm{Zr}$ & SnO2 & $\begin{array}{l}\text { TiOxNy top } \\
\text { layer }\end{array}$ \\
\hline 3803 & $200 \mathrm{~nm}$ & & & & & \\
\hline 3807 & $20 \mathrm{~nm}$ & & & & & \\
\hline 3806 & $20 \mathrm{~nm}$ & $200 \mathrm{~nm}$ & & & & \\
\hline 3808 & $20 \mathrm{~nm}$ & $10 \mathrm{~nm}$ & & & & \\
\hline 3811 & $20 \mathrm{~nm}$ & $10 \mathrm{~nm}$ & $10 \mathrm{~nm}$ & $5 \mathrm{~nm}$ & $200 \mathrm{~nm}$ & \\
\hline 3810 & $20 \mathrm{~nm}$ & $10 \mathrm{~nm}$ & $10 \mathrm{~nm}$ & $5 \mathrm{~nm}$ & $40 \mathrm{~nm}$ & \\
\hline 3814 & $20 \mathrm{~nm}$ & $10 \mathrm{~nm}$ & $10 \mathrm{~nm}$ & $5 \mathrm{~nm}$ & $40 \mathrm{~nm}$ & $200 \mathrm{~nm}$ \\
\hline 3813 & $20 \mathrm{~nm}$ & $10 \mathrm{~nm}$ & $10 \mathrm{~nm}$ & $5 \mathrm{~nm}$ & $40 \mathrm{~nm}$ & $30 \mathrm{~nm}$ \\
\hline
\end{tabular}

\subsection{Nanoindentation testing}

Nanoindentation testing of the coated samples was carried out using a Hysitron Triboindenter fitted with a new Berkovich tip (tip end radius 150nm) and compared to data obtained from float glass using an older used Berkovich tip with a larger tip end radius ( $200 \mathrm{~nm})$. Measurements were made at a range of peak contact loads from $1 \mathrm{mN}$ to $100 \mu \mathrm{N}$ starting from the highest loads and finishing with the lowest to ensure that the machine noise and thermal drift was minimised for the most sensitive measurements. Prior to testing the tip end shape and machine compliance was carefully calibrated using the method of Oliver and Pharr [12]. Hardness and contact modulus were determined from the load displacement curves using the Oliver and Pharr approach. The elastic properties of the thick coatings were determined using the ISO14577 extrapolation method [7] and compared to fitted values determined from the model of Bull for a single layer coating [4]. The fitted values were then optimised by using a full multilayer analysis described in the next section.

\subsection{Simple model for the contact modulus of a multilayer coating}

The model of Bull [4] is based on a truncated cone of load support beneath the indenter and the displacements in any coating layers can be calculated if their thickness and elastic properties are known. If we assume a totally rigid indenter then the indenter displacement, $\delta$, is given by the sum of all the displacements in the individual layers beneath the indenter. For a simple coating on a substrate it can be shown that the indenter displacement in the coating is given by

$\delta_{c}=\frac{P}{\pi E_{c}}\left[\frac{1}{a_{0} \tan \alpha}-\frac{1}{a_{0} \tan \alpha+t_{c} \tan ^{2} \alpha}\right]$

where $a_{0}$ is the contact radius, $t_{c}$ is the coating thickness and $\alpha$ is the cone angle. For the substrate we have 
$\delta_{s}=\frac{P}{\pi E_{s}}\left[\frac{1}{a_{0} \tan \alpha+t_{c} \tan ^{2} \alpha}-\frac{1}{a_{0} \tan \alpha+\left(t_{c}+t_{s}\right) \tan ^{2} \alpha}\right]$

where $E_{\mathrm{s}}$ is the Young's Modulus of the substrate and $t_{\mathrm{s}}$ the substrate thickness. The total indenter displacement is then

$\delta=\delta_{c}+\delta_{s}$

Both the displacements in the coating and substrate are a linear function of contact load and thus the unloading stiffness $S=P / \delta$. Given that for a flat punch $S=2 E a[13]$ it is possible to calculate the effective Young's Modulus of the coating/substrate system

$E=\frac{P}{2 a_{0}\left(\delta_{c}+\delta_{s}\right)}$

For a deformable indenter $E$ must be replaced by $E^{*}$, the contact modulus, given by

$\frac{1}{E^{*}}=\frac{1-v_{1}^{2}}{E_{1}}+\frac{1-v_{2}^{2}}{E_{2}}$

where the subscripts 1 and 2 refer to the properties of the sample and indenter respectively and $v$ is Poisson's ratio and $E$ is Young's Modulus. For a coated system, $E_{\mathrm{c}}$ and $E_{\mathrm{s}}$ are the contact moduli of coating and substrate respectively. The contact radius can be related to the contact depth, $h_{\mathrm{c}}$, determined by the Oliver and Pharr method [12], if the indenter geometry is known. For a Berkovich indenter,

$h_{c}=\sqrt{(\pi / k)} a_{0}$

where $k=24.5$. This assumes a perfect tip which is reasonable at larger contact areas corresponding to higher load tests. When comparing with experimental data it should be recognised that the experimental contact depth will have been used to determine a contact area through a measured tip area function - thus for a given contact depth there could be a range of contact moduli depending on the area function; usually lower values are measured for a blunter tip. To avoid this problem the contact radius can be determined from the contact area, $A$, determined from the contact depth using the tip area function, via

$a_{0}=\sqrt{A / \pi}$

Or more conveniently from the load and measured hardness

$a_{0}=\sqrt{\frac{P}{\pi H}}$

Since the indentation of a bulk material is almost identical to that of a coating with large thickness equation (1) can be applied to a bulk material of known elastic properties to determine the truncated cone angle $\alpha$. If we assume that the material thickness is very much greater than the contact radius the second term in the brackets in equation (1) goes to zero and

$\delta=\frac{P}{E \pi a_{0} \tan \alpha}$

The unloading stiffness is $P / \delta$ and using the Sneddon approximation [13] 
$S=E \pi a_{0} \tan \alpha=2 E a_{0}$

Thus $\tan \alpha=2 / \pi$ and $\alpha=32.48^{\circ}$. This angle is independent of the choice of material and the properties of coating or substrate. Combining equations (1), (2) and (4) with this expression for tan $\alpha$ it can easily be shown that:

$E=\frac{1}{\frac{1}{E_{C}}\left[1-\frac{\pi a_{0}}{\pi a_{0}+2 t_{C}}\right]+\frac{1}{E_{S}}\left[\frac{\pi a_{0}}{\pi a_{0}+2 t_{C}}-\frac{\pi a_{0}}{\pi a_{0}+2\left(t_{C}+t_{S}\right)}\right]}$

From this formulation it is clear that as $a_{0}$ tends to zero the value of $E$ tends to $E_{\mathrm{c}}$ and that if $a_{0}$ is very much greater than $t_{\mathrm{c}}$ and $t_{\mathrm{s}}$ is much greater than $a_{0}$ then $E$ tends to $E_{\mathrm{s}}$ as might be expected.

Equations (1)-(4) can easily be modified for a coating with two or more layers. In the case of a layer on an intermediate layer on the substrate equation (1) remains valid for the coating contribution but, for an interlayer of thickness $t_{i}$ and modulus $E_{i}$, equation (2) becomes

$\delta_{i}=\frac{P}{\pi E_{i}}\left[\frac{1}{a_{0} \tan \alpha+t_{c} \tan ^{2} \alpha}-\frac{1}{a_{0} \tan \alpha+\left(t_{c}+t_{i}\right) \tan ^{2} \alpha}\right]$

And for the substrate

$\delta_{S}=\frac{P}{\pi E_{S}}\left[\frac{1}{a_{0} \tan \alpha+\left(t_{c}+t_{i}\right) \tan ^{2} \alpha}-\frac{1}{a_{0} \tan \alpha+\left(t_{c}+t_{i}+t_{s}\right) \tan ^{2} \alpha}\right]$

In equations (3) and (4) $\delta_{\mathrm{c}}+\delta_{\mathrm{s}}$ is replaced by $\delta_{\mathrm{c}}+\delta_{\mathrm{f}}+\delta_{\mathrm{s}}$. Thus

$E=\frac{1}{\frac{1}{E_{C}}\left[1-\frac{\pi a_{0}}{\pi a_{0}+2 t_{c}}\right]+\frac{1}{E_{i}}\left[\frac{\pi a_{0}}{\pi a_{0}+2 t_{C}}-\frac{\pi a_{0}}{\pi a_{0}+2\left(t_{C}+t_{i}\right)}\right]+\frac{1}{E_{S}}\left[\frac{\pi a_{0}}{\pi a_{0}+2\left(t_{C}+t_{i}\right)}-\frac{\pi a_{0}}{\pi a_{0}+2\left(t_{c}+t_{i}+t_{S}\right)}\right]}$

The denominator of this equation is the sum of the reciprocals of the elastic moduli of each individual layer multiplied by a geometric factor which depends on the contact radius and individual coating and substrate thicknesses. In fact the thickness contributions in each term represent the distance of the top and bottom of the individual layer from the sample surface. This approach can easily be generalised to a multilayer coating with more than two layers.

\section{Results and discussion}

\subsection{Measurements on uncoated float glass}

Figure 1 shows measurements of the contact modulus as a function of contact scale for fused silica and the two sides of float glass. As expected the fused silica does not show a variation with contact depth since the tip calibration is good down to contact depths of less than $25 \mathrm{~nm}$. However, for both sides of float glass the contact modulus increases as the contact scale is reduced. In this study the tin side shows a greater modulus than the air side in contrast to what was observed previously by Derby et al [14] but in agreement with the increased stiffness of tin doped glass reported by Krohn et al [15]. 


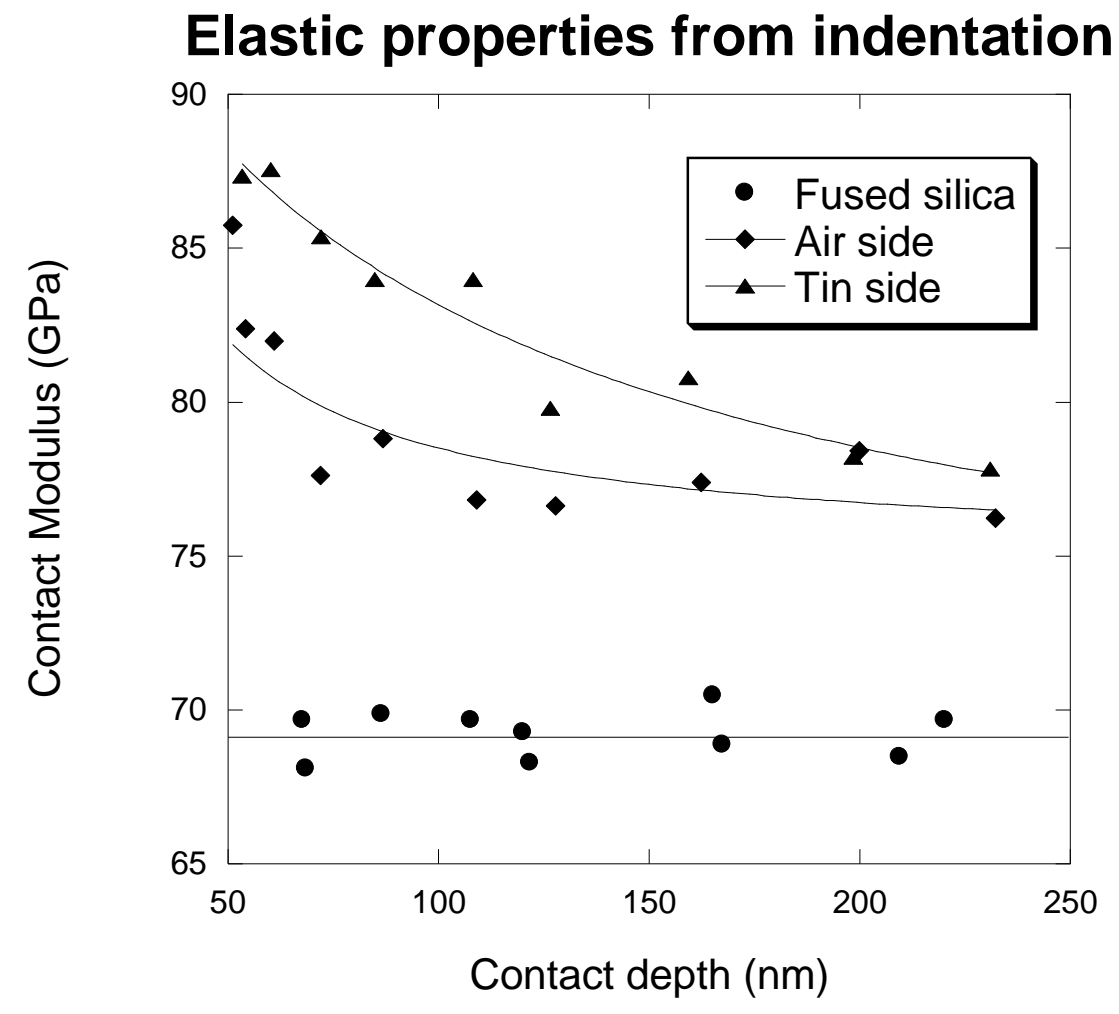

Figure 1: Variation of contact modulus with contact depth for fused silica and the two sides of float glass.

To get good data for the coatings this variation in contact modulus needs to be taken into consideration in any modelling. The data for the air side has been investigated further since all coatings were deposited on the air side in this study. There are a number of ways of modelling the experimental data to produce a curve with a reasonable fit to the experimental data including a single surface layer of $1 \mu \mathrm{m}$ thickness and contact modulus of $81 \mathrm{GPa}$ on a glass substrate with contact modulus of 73GPa (the contact modulus of the glass determined from bending tests), a $200 \mathrm{~nm}$ layer with contact modulus of $85 \mathrm{GPa}$ on a $200 \mathrm{~nm}$ layer with contact modulus of $81 \mathrm{GPa}$ on a 73GPa glass substrate and a graded coating with contact moduli reducing from $90 \mathrm{GPa}$ to $80 \mathrm{GPa}$ at $1 \mathrm{GPa} / 50 \mathrm{~nm}$ on the base glass (Figure 2). In all cases the stiffer layer has substantial thickness. For the purposes of modelling the coated system a $1 \mu \mathrm{m}$ layer with contact modulus $81 \mathrm{GPa}$ on a glass substrate with contact modulus 73GPa was adopted as being most reliable - the rapid increase in measured modulus at low penetration is probably due to incomplete plasticity at the elastic-plastic transition leading to inaccuracies in the contact areas and hence elastic properties derived by the Oliver and Pharr method [12] used in this study. However, the increase in stiffness in the surface region may also be a result of different microstructure caused by a combination of more rapid surface cooling and leaching of glass modifiers during glass manufacture. 


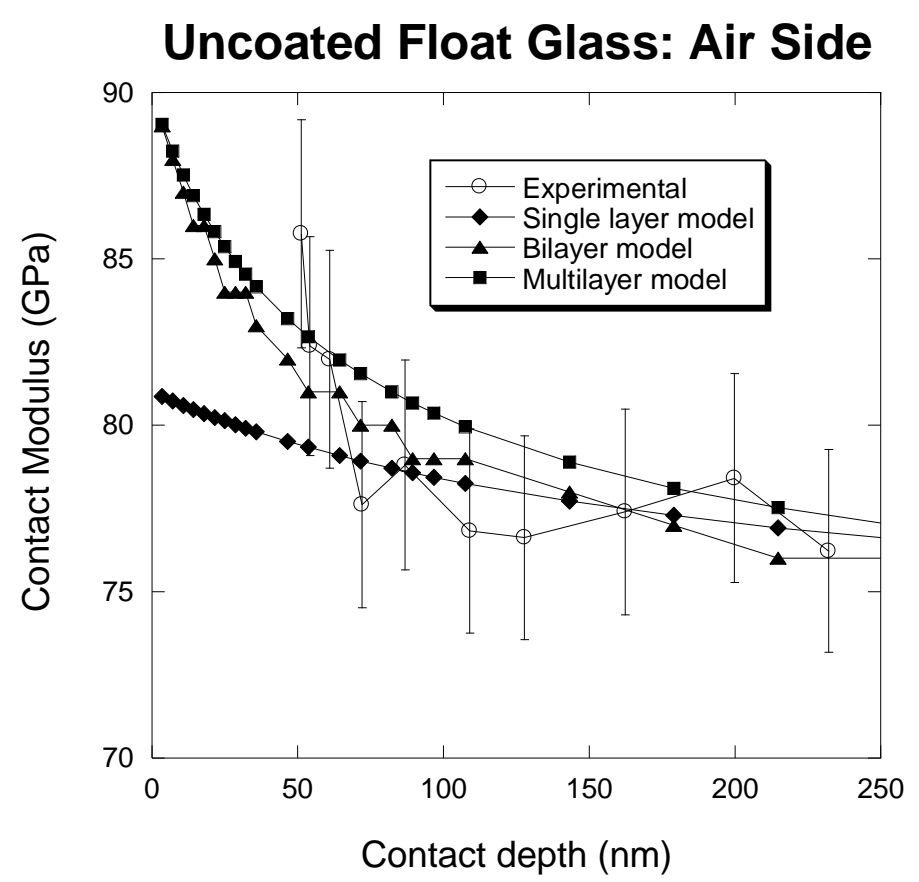

Figure 2: Modelled variation of contact modulus with contact depth compared with experimental data for the air side of float glass. $A 1 \mu \mathrm{m}$ single layer of $E=83 \mathrm{GPa}$ on a glass substrate $(E=73 \mathrm{GPa}$ ) is compared with a bilayer coating $200 \mathrm{~nm} E=85 \mathrm{GPa}$ on $1 \mu \mathrm{m} \mathrm{E}=81 \mathrm{GPa}$ on glass and a multilayer coating where the modulus is graded from $90 \mathrm{GPa}$ to $80 \mathrm{GPa}$ in $200 \mathrm{~nm}$ layers.

\subsection{Thick vs thin coatings}

Figures $3 a$ and $b$ show a comparison of the different oxide top coat materials at 200nm thickness (thick) and optical design thickness (thin). Data for the uncoated air side of float glass is included for comparison. There is a distinct increase in the measured moduli due to the presence of the coating for both the thicker and thinner layers though the glass substrate dominates measurements for the thinner layers. At contact depths less than $70 \mathrm{~nm}$ there is a rapid increase in contact modulus and the coated and uncoated results converge. This confirms that the data in this region is controlled by the elastic-plastic transition and is not very reliable for use in assessment of coating properties unless care is taken to ensure that the transition to plasticity has occurred. Between 70 and $200 \mathrm{~nm}$ contact depth the enhanced stiffness of the coatings increases the measured contact modulus over that of the uncoated sample but above $200 \mathrm{~nm}$ the coated and uncoated data converge as the substrate dominates the measured data.

For the thin coatings (Figure 3a) there is a minimum in the measured contact modulus at $70 \mathrm{~nm}$ contact depth and a maximum at $130 \mathrm{~nm}$. This is observed for all the coatings tested here but not in the original float glass measurement. The variation is the same for all coatings and is most likely due to inaccuracies in the tip area function used in the property calculations. The float glass was tested with a used tip and shows the expected behaviour when the tip calibration is good and the low load data is affected by the elastic-plastic transition. The coatings were tested with a different brand new tip which was much sharper and was able to generate plastic deformation and lower loads (and 
hence contact depths). This tip was calibrated according to the method of Oliver and Pharr [12] immediately before the tests were carried out but the calibration is not a perfect description of the tip profile. This minimum and maximum behaviour (with a less than $5 \%$ variation) is associated with a truncated tip where there is a sharp transition from the flattened end to the sloping sides of the indenter. The Oliver and Pharr tip area function is designed to fit smoothly varying tip profiles and, when there is a discontinuity, will not match the tip profile exactly after the fitting process. In fact in the transition region the fit will generally underestimate the contact area resulting in an overestimate of the contact modulus, hence the maximum observed for the coatings in Figure $3 a$. Once the tip has been used for some time the edges of the truncated region become rounded and the Oliver and Pharr tip area function is a good description of the tip shape.

Figure 4 shows the ISO14577 extrapolated values for the contact moduli of the coatings determined for the $200 \mathrm{~nm}$ layers. Only data obtained from indentations where there was a significant plastic work of indentation (i.e. the loading and unloading curves were not coincident) and a well-defined impression was observed in post facto AFM by the tip which made the test were used in the extrapolation. This ensures that sufficient plasticity had occurred to make the extrapolations valid. The values are within the range of what has been measured previously [e.g. 16-18]. The properties of the two different TiOxNy layers are different despite being deposited using the same process parameters. This highlights the importance of the layer onto which a coating is deposited; the structure of this underlayer can affect the nucleation and growth of the coating subsequently deposited and therefore its microstructure and properties.

(a)

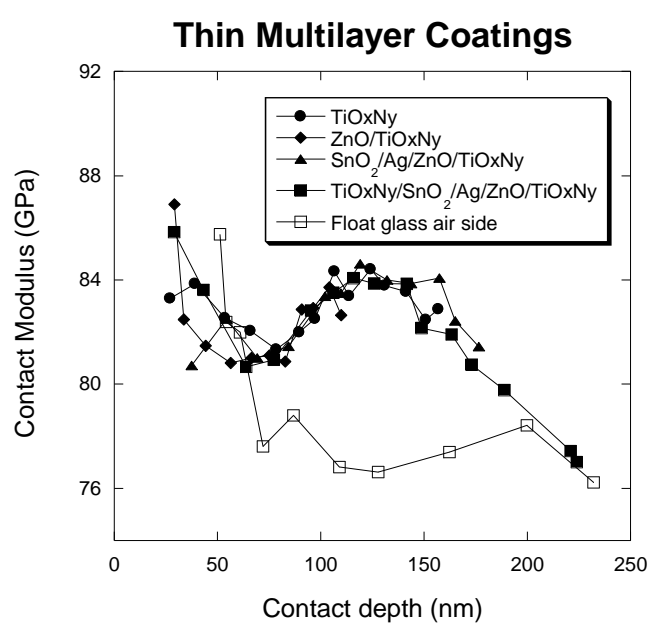

(b)

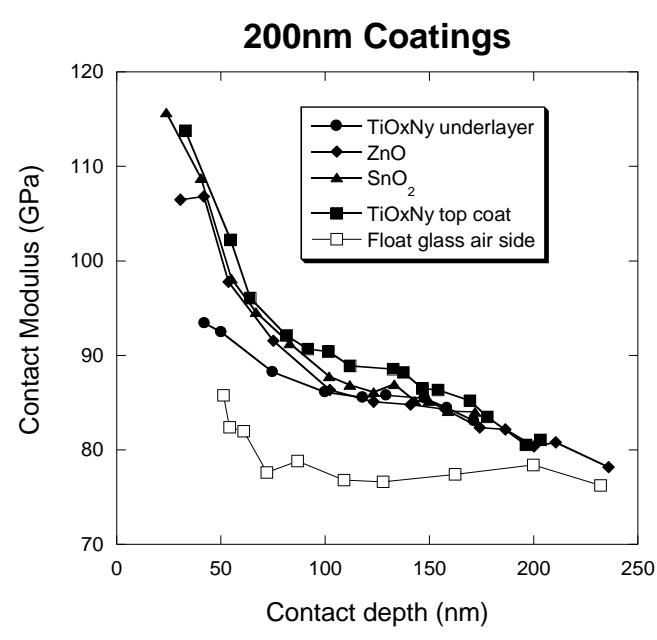

Figure 3: Variation of experimentally measured contact modulus with contact depth for a range of coated samples compared to data obtained from the uncoated air side of float glass. (a) thin coatings and (b) $200 \mathrm{~nm}$ thick coatings. 


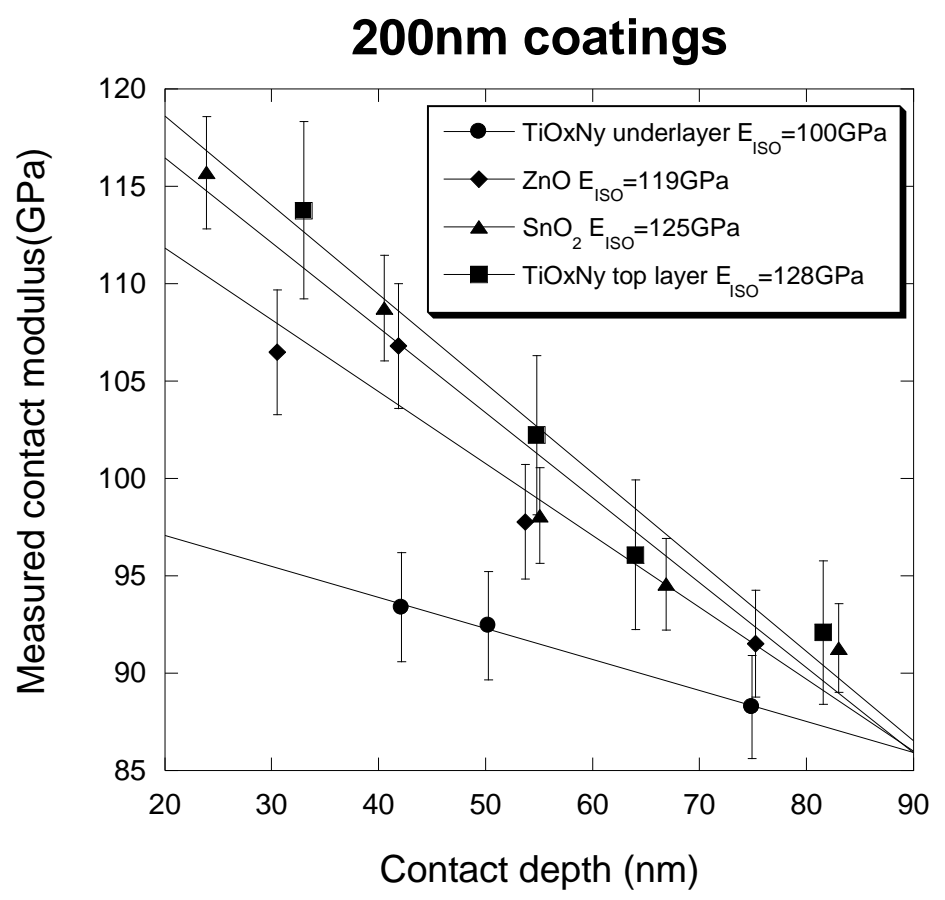

Figure 4: ISO14577-Part 4 extrapolations to determine the contact modulus of the $200 \mathrm{~nm}$ thick oxide coatings in this study.

\subsection{Modelling}

\subsubsection{Does the ISO14577 extrapolation produce good coating data?}

Contact modulus data for the $200 \mathrm{~nm} \mathrm{ZnO}$ coating has been modelled in a number of different ways to compare with experimental data in Figure 5. The bilayer and multilayer fitting was done using equations (11) and (14) using the measured contact modulus and depth data as input and the individual layer moduli as fit parameters. In the case of the single layer model the ISO14577 extrapolated contact modulus, E=119GPa, has been used and the substrate contact modulus has been set to $81 \mathrm{GPa}$. The modelled curve fits the experimental data above $100 \mathrm{~nm}$ contact depth but underestimates it at lower depths. The bilayer model attempts to include a better description of the substrate properties by treating the substrate as a $1 \mu \mathrm{m} 81 \mathrm{GPa}$ layer on top of a 73GPa substrate. With a $200 \mathrm{~nm} \mathrm{ZnO}(\mathrm{E}=119 \mathrm{GPa})$ top layer the predicted variation of contact modulus with contact depth is almost identical to the single layer model but gives slightly better agreement with experimental data in the $50-70 \mathrm{~nm}$ contact depth range. To get the best fit across the complete range of data a multilayer model was constructed consisting of 200nm ZnO ( $E=140 \mathrm{GPa}$ ) on 20nm TiOxNy ( $E=105 \mathrm{GPa}$ ) on a glass substrate modelled as two layers ( $1 \mu \mathrm{m} \mathrm{E}=81 \mathrm{GPa}$ on a 73GPa substrate). The fit is good at low and high contact depth but overestimates the contact modulus in the $70-200 \mathrm{~nm}$ contact depth range where the experimental data is most reliable. Given the errors in the experimental data it is not possible to reliably separate the behaviour of the different models. 


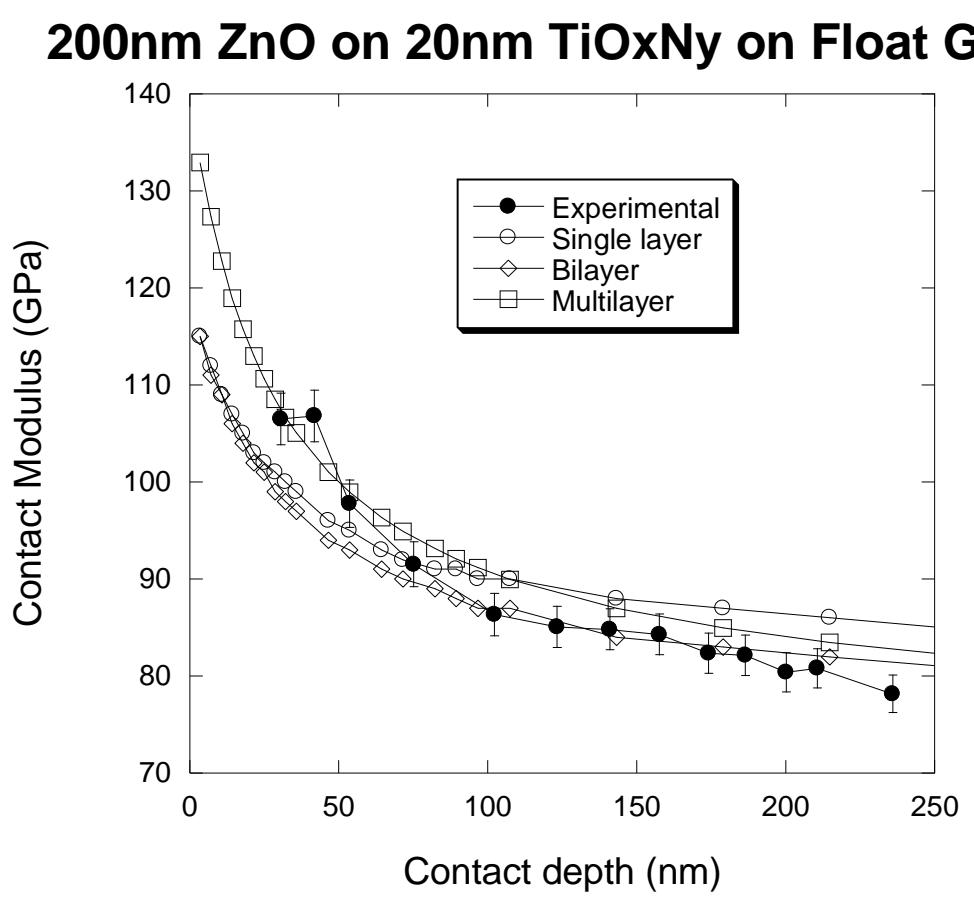

Figure 5: Variation of contact modulus with contact depth for $200 \mathrm{~nm} \mathrm{ZnO}$ on $20 \mathrm{~nm}$ TiOxNy on the air side of float glass. The behaviour has been modelled as a single layer coating, a bilayer coating where the substrate is considered as a $1 \mu \mathrm{m} 81 \mathrm{GPa}$ contact modulus layer on a 73GPa foundation and as the full multilayer with the same structure as the bilayer but including a 20nm TiOxNy layer underneath the $\mathrm{ZnO}$.

\subsubsection{Optimised fitting parameters for thin and thick coatings}

Given that the substrate behaviour is well described by a two layer model the multilayer models can be optimised to give the best fit to experimental data in the $70-200 \mathrm{~nm}$ contact depth range to determine coating properties. This has been done by starting at the layer closest to the glass and then using the optimised coating properties for the underlayers of the subsequent coatings. Results of the fitting are shown in Figure 6 for the 200nm thick coatings. The optimised and ISO14577 extrapolated values for coating properties are compared in Table 2. Very good fits are produced in all cases. The fitted values for the contact modulus are usually greater than the ISO 14577 extrapolations. 
(a)

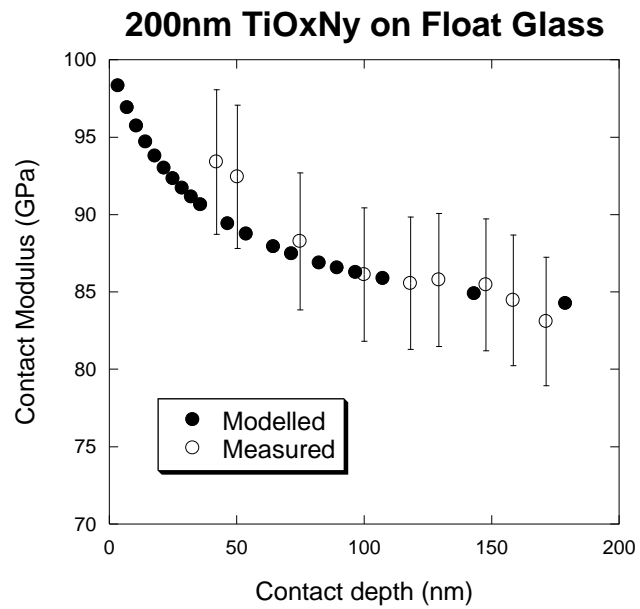

(c)

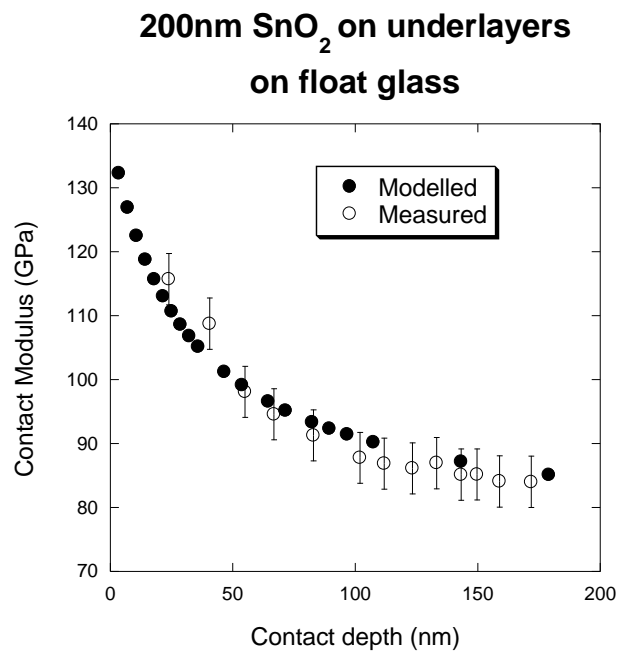

(b)

200nm ZnO on 20nm TiOxNy on Float Glass

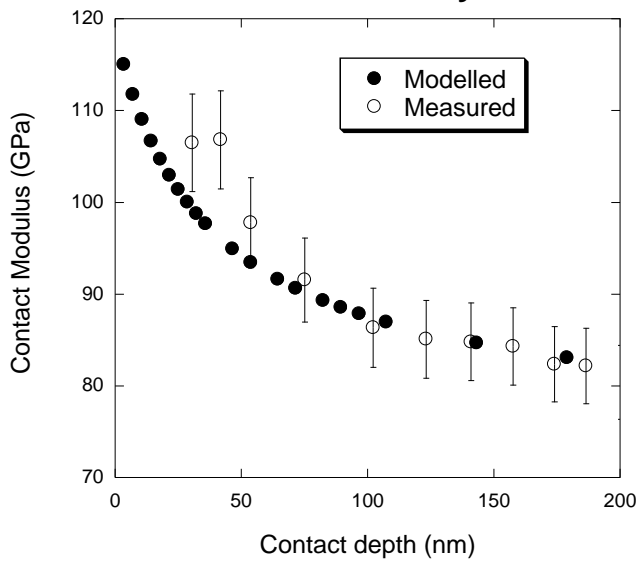

(d)

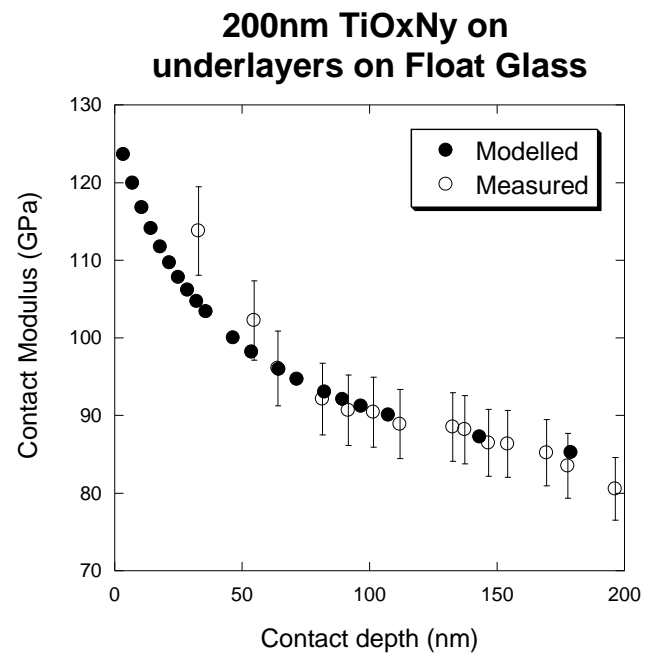

Figure 6: Optimised fits for contact modulus as a function of contact depth for thick (200nm) coatings on correct underlayers with solar control coating thickness on the air side of float glass. (a) TiOxNy underlayer, (b) $\mathrm{ZnO}$, (c) $\mathrm{SnO}_{2}$ and (d) TiOxNy top layer.

The fitting data in Table 2 was then used to predict the contact modulus versus contact depth behaviour for the thinner coatings with the same thickness as in solar control coatings and the comparison with experimental data is shown in Figure 7. The fit to the data for the TiOxNy underlayer is relatively poor in the greater than $100 \mathrm{~nm}$ contact depth range and the measured contact modulus is underestimated. However, the modulus of this layer is low compared to that of the top TiOxNy for the thicker coatings and this suggests that the 200nm TiOxNy underlayer was not as dense as expected. For the other coatings the predictions diverge at the lowest contact depths where the model overestimates the measured data. Again this could be due to lower density coatings when deposited to thicknesses of 10 s of nanometres. 
(a)

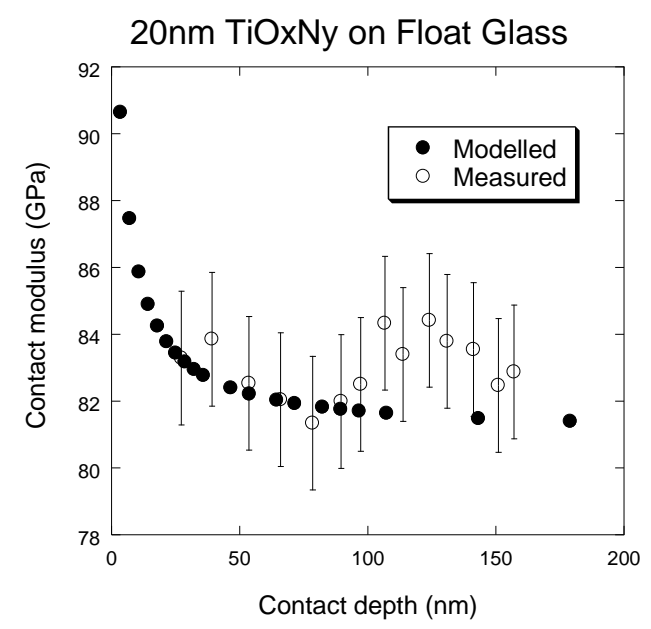

(c)

$40 \mathrm{~nm} \mathrm{SnO}{ }_{2}$ on $10 \mathrm{~nm} \mathrm{Ag}$ on $10 \mathrm{~nm} \mathrm{ZnO}$ on $20 \mathrm{~nm}$ TiOxNy on Float Glass

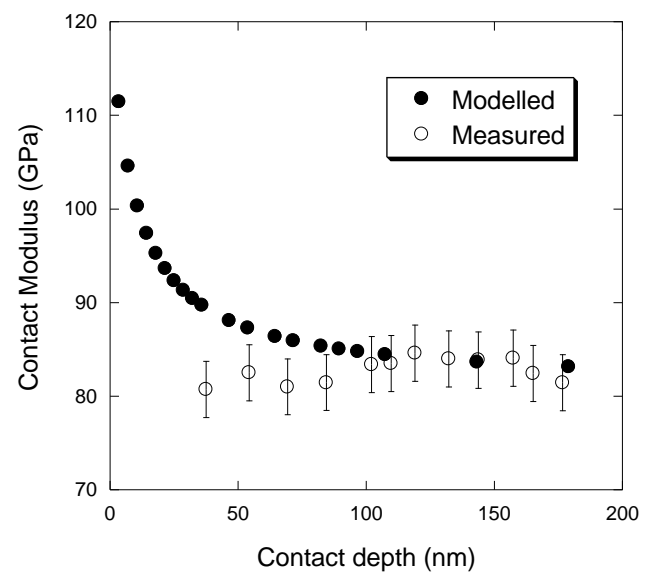

(b)

10nm ZnO on 20nm TiOxNy on Glass

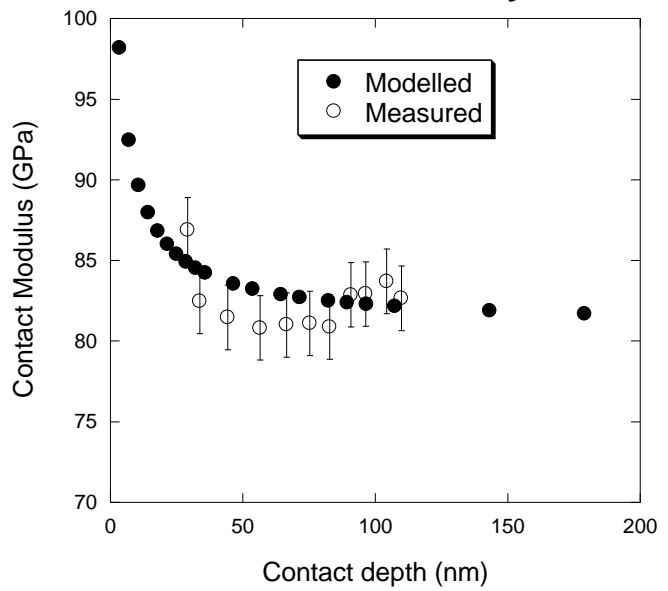

(d)

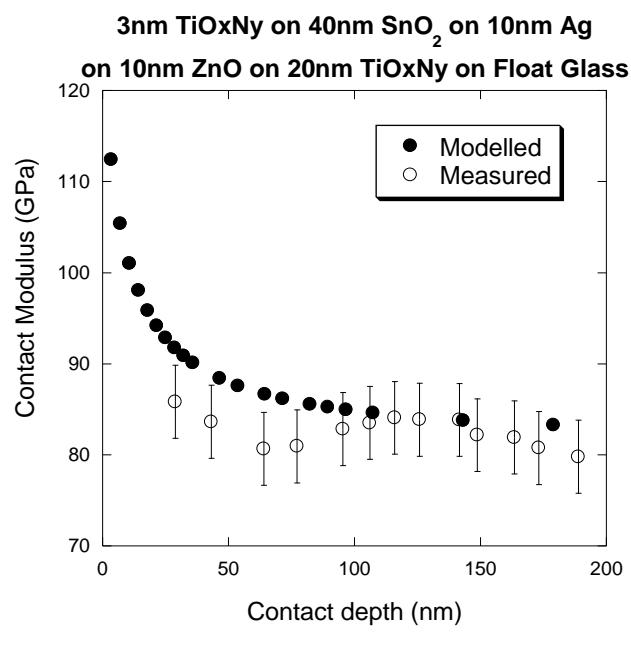

Figure 7: Predicted variation of contact modulus as a function of contact depth for thin multilayer coatings with solar control coating thickness on the air side of float glass. The multilayer stack stops at (a) TiOxNy underlayer, (b) $\mathrm{ZnO}$, (c) $\mathrm{SnO}_{2}$ and (d) TiOxNy top layer. 
Table 2: Comparison of contact moduli of coatings from the ISO14577 extrapolation method and from optimisation using the simple model outlined in this study

\begin{tabular}{|l|c|c|}
\hline Coating Material & $\begin{array}{l}\text { ISO 14577 Part 4 contact } \\
\text { modulus from extrapolation } \\
\text { (GPa) }\end{array}$ & $\begin{array}{l}\text { Contact modulus from model } \\
\text { optimisation (GPa) }\end{array}$ \\
\hline TiOxNy underlayer & 100 & 105 \\
\hline $\mathrm{ZnO}$ & 119 & 140 \\
\hline $\mathrm{SnO}_{2}$ & 125 & 145 \\
\hline $\mathrm{TiOxNy}$ top layer & 128 & 136 \\
\hline
\end{tabular}

A low value of contact modulus is often observed when using the extrapolation method if the coating thickness is low since the substrate properties will dominate the measured data $[19,20]$. For practical purposes the oxide coating thickness should be at least $400 \mathrm{~nm}$ if coatings on glass are to be assessed by the extrapolation method. Another area of concern is the radius of the tip used in the measurement [21]. The tip radius should be less than $10 \%$ of the coating thickness if the measured contact modulus is to be unaffected by tip rounding. This is not achieved in this study and the increase in measured contact modulus at low contact depth is thus likely to be an artefact of tip end shape.

\section{Conclusions}

The elastic properties of thin oxide coatings on glass have been assessed by a combination of careful nanoindentation testing and modelling. The contact modulus depends on the structure of the coatings which in turn depend on the underlying layer structure in a multilayer coating. The properties of the substrate need to be understood if the properties of the coating are to be determined accurately; in this study the air side of float glass shows a surface layer which is stiffer than the bulk and influences nanoindentation measurements. When thin oxide coatings on glass are tested the ISO14577 extrapolation method will underestimate the contact modulus of glass and a coating thickness of $400 \mathrm{~nm}$ or greater is needed to get reliable data using this method. A simple model for contact modulus as a function of contact depth has been extended to analyse multilayer coatings and an optimisation approach used to establish reasonable elastic property values for the coatings in a multilayer stack.

\section{Acknowledgements}

The author would like to thank Eva Gutierrez-Berasetegui and Jinju Chen for the provision of experimental data and useful discussions. John Ridealgh and Paul Warren of Pilkington plc are also thanked for provision of samples and funding part of the work. 


\section{References}

[1] S. D. Gupta and G. S. Agarwal, Opt. Express 15 (2007) 9614-9624.

[2] S.S. Kanu and R. Binions, Proc. Roy. Soc., A, 466 (2010) 19-44.

[3] K.J Belde and S.J Bull. Journal of Adhesion Science and Technology, 22 (2008) 121-132.

[4] S.J. Bull, Phil. Mag., (2014); DOI:10.1080/14786435.2014.909612.

[5] S.J. Bull, J. Phys. D: Applied Physics, 38 (2005) R393-R413.

[6] J. Chen and S.J. Bull, Vacuum, 83 (2009) 911-920.

[7] ISO14577 Part 4 (2007) Metallic materials -- Instrumented indentation test for hardness and materials parameters -- Part 4: Test method for metallic and non-metallic coatings, ISO, Geneva, Switzerland.

[8] C. Zeibert and S. Ulrich, J. Vac. Sci. Technol., A24 (2006) 554-583.

[9] Y.T. Pei, G.M Song, W. Sloof. And J. Th. M. de Hosson, Surf. Coat. Technol., 201 (2007) 69116916.

[10] A. Tricoteaux, G. Duarte, D. Chicot, E. Le Bourhis, E. Bemporad and J. Lesage, Mechanics of Materials, 42 (2010) 166-174.

[11] Smithells Metals Reference Book, Eds. W.F. Gale and T.C. Totmeier, $8^{\text {th }}$ Edition, ButterworthHeinemann Ltd, 2003.

[12] W.C. Oliver and G.M. Pharr, J. Mater. Res., 7 (1992) 1564-1583.

[13] I.N. Sneddon, Int. J. Eng. Sci., 3 (1965) 47-57.

[14] O. Goodman and B. Derby, Acta Materialia, 59 (2011) 1790-1799.

[15] M.H. Krohn, J.R. Hellman, C.G. Pantano, M.G. Lower and R.K. Brow, Fracture Mechanics of Ceramics 8: Active Materials, Nanoscale Materials, Composites, Glass, and Fundamentals, Eds. R.C. Bradt, D. Munz, M.Sakai and K.W. White, Springer, New York, (2005), pp135-148.

[16] P. Soares, A. Mikowski, C.M. Lepienski, E. Santos Jr, G.A. Soares, V. Swinka Filho and N.K. Kuromoto, J Biomed Mater Res B: Appl Biomater. 84 (2008) 524-530.

[17] T.-H. Fang, W.-J. Chang, and C.-M. Lin, Materials Science and Engineering A, 452-453, (2007) 715-720.

[18] P. Lemoine, P. Mariotti and D. Maguire, Thin Solid Films, 401 (2001) 196-202.

[19] S.J. Bull, J. Vac. Sci. Technol., A30, (2012) 01A1690.

[20] S.J. Bull, Thin Solid Films, 571 (2014) 290-295.

[21] A.M. Korsunsky and A. Constantinescu, Thin Solid Films, 517 (2009) 4835. 